ISSN 1112-9867

\title{
OPTIMIZATION OF CONDITIONS FOR IN VITRO POLLEN GERMINATION AND POLLEN TUBE GROWTH OF DATE PALM (PHOENIX DACTYLIFERA L.)
}

\author{
M. Mesnoua ${ }^{1}$, M. Roumani ${ }^{1}$, M. K. Bensalah ${ }^{1}$, A. Salem ${ }^{1}$, A. Benaziza ${ }^{2}$ \\ ${ }^{1}$ Division Phoeniciculture, Centre de Recherche Scientifique et thechnique sur les Régions \\ Arides, Biskra, BP 1682 R.P. Biskra 07000, Algeria \\ ${ }^{2}$ Université Mohamed Khider. Biskra, Algérie
}

Received: 23 August 2017/ Accepted: 25 November 2017 / Published online: 01 January 2018

\begin{abstract}
Pollen germination and pollen tube growth might have a significant effect on fruit and seed production. This study was conducted to investigate the best medium for pollen germination and pollen tube growth of date palm male. Significant differences in percentages of pollen germination and pollen tube growth were observed between mediums. The use agar-based medium containing $5 \%$ of sucrose, $0.20 \mathrm{~g} / 1$ boric acid, $0.42 \mathrm{~g} / 1$ calcium nitrate, $0.1 \mathrm{~g} / 1$ potassium nitrate and $0.22 \mathrm{~g} / 1$ magnesium sulfate gave the best results in terms of germination and/or tube growth. The germination percentage was declined with polyethylene glycol and naphthaleneacetic acid, and inhibited completely with fructose, glucose and date extract. These results could have implications not only for optimizing pollen management in date palm but also to select the best pollinizer for this specie.
\end{abstract}

Keywords: In vitro pollen germination; Sucrose; Fructose; Glucose; Naphthaleneacetic acid; basal medium.

Author Correspondence, e-mail: mohamesnoua@gmail.com

doi: $\underline{\text { http://dx.doi.org/10.4314/jfas.v10i1.11 }}$

\section{INTRODUCTION}

Le palmier dattier (Phoenix dactylifera L.) est considéré comme étant l'arbre de vie dans les régions sahariennes des pays de l'Afrique du Nord et du Moyen-Orient. En 
effet, cette arbre dioïque et pérenne, constitue l'axe principal de l'agro-système oasien (1,2). L'Algérie est un pays phoenicicole classé au troisième rang mondial, selon les statistiques de la FAO 2015, avec une production d'environ 1 million tonnes/an (3). Cependant, cette production reste relativement faible vue la grande surface à vocation phénicicole de ce payé.

Pour assurer une meilleure production, les phoeniciculteurs pratiquent la pollinisation manuellement en choisissant le pollen de bonne qualité. Le type du pollen et la pollinisation efficace ont des effets importants sur la qualité des fruits et la production de semences dans la plupart des plantes $(4,5)$. Par conséquent, l'étude de la biologie du pollen, y compris la viabilité et la croissance du tube pollinique, sont nécessaires pour toute approche rationnelle pour augmenter la productivité (47). La germination in vitro et la longueur du tube pollinique sont des paramètres très importants pour tester la viabilité du pollen (8). La viabilité du pollen peut être évaluée soit par des techniques de coloration ou par des tests de germination in vitro et in vivo (4). Dans de nombreuses espèces, la germination du pollen in vitro dépend de l'ajout de substrats clés au milieu de culture tels que le nitrate de calcium et l'acide borique (9).

L'objectif de ce travail est de développer un milieu de culture optimal pour la germination des grains du pollen et la croissance du tube pollinique de palmier dattier.

\section{MATERIEL ET METHODES}

Ce travail a été au laboratoire de la culture des tissus de palmier dattier situé à l'Outaya (CRSTRA). Le pollen est collecté à partir d'un arbre mâle âgé de 10 ans, dont les caractéristiques phénotypiques sont similaires au cultivar Moch degla. Les spathes sont séparés de leurs enveloppes, et laissés déshydrater à température ambiante dans un endroit obscure pendant une semaine jusqu'à la déhiscence des anthères. Le pollen de cinq spathes est extrait en secouant les fleurs déhiscentes, et mélangé pour minimiser les variations qui pourraient exister entre les différents spathes.

\section{Effet du milieu de culture sur la germination des grains de pollen et la longueur du tube pollinique}


Pour la compostion minérale, nous avons utilisé un milieu de base (MB) développé pour le pollen de l'avocatier (7). Ce milieu est composé de 0,42 g/l nitrate de calcium $\mathrm{Ca}(\mathrm{NO} 3) 2, \quad 0,20 \mathrm{~g} / 1$ acide borique $\mathrm{H} 3 \mathrm{BO} 3,0,1 \mathrm{~g} / 1$ nitrate de potassium KNO3 et 0,22 g/l sulfate de magnésium MgSO4.7H2O. A ce milieu nous avons ajouté comme variables les composés suivants : sucrose $20 \%$, glucose $20 \%$, fructose $20 \%$, jus de dattes $30 \%$ (cultivar deglat beida), polyéthylène glycol $10 \%$ (PEG) et acide naphtalène acétique (ANA) 4mg/l. Le témoin est composé par le MB seulement. Après avoir déterminé le milieu optimal pour la germination du pollen qui est le sucrose $20 \%$ additionné au MB, nous avons déterminé dans une autre expérimentation la concentration optimale du sucrose, nous avons utilisé six doses: $5-10-15-20-25$ et $30 \%$. Les différents milieux de culture sont solidifiés par l'agar $1 \%$ et autoclavés pendant $15 \min$ à $121^{\circ} \mathrm{C}$ avant leurs utilisations.

Les grains de pollen sont dispersés uniformément sur le milieu de culture, et ensuite incubées à l'obscurité à $25^{\circ} \mathrm{C}$ pendant 24 heures. Pour chaque traitement, le pourcentage moyen de la germination est calculé à partir de 6 champs de vision, et la longueur moyenne des tubes pollinique à partir de 20 tubes polliniques. Un grain de pollen est considéré comme ayant germé lorsque la longueur du tube pollinique soit supérieure ou égale au diamètre de la grain (10). Les grains de pollen sont photographiés à l'aide d'un microscope optique (optika) muni d'une caméra, et les différentes mesures sont effectuées à l'aide d'un logiciel (Opmias Ver1.0).

\section{Analyse statistique}

Toutes les analyses statistiques ont été réalisées avec le logiciel SPSS version 21. Les données expérimentales ont été initialement testées pour la normalité et l'homogénéité de la variance, afin de répondre aux exigences statistiques. Les données ont été exprimées en moyenne \pm écart-type (SD). Le traitement statistique des données a été effectuée en utilisant une analyse de variance (ANOVA) et le test de Tukey au seuil $\mathrm{P}<0,05$ a été utilisé pour la comparaison des moyennes.

\section{RESULTATS ET DISCUSSION}

Afin de développer un milieu de culture optimal pour la germination des grains du pollen de palmier dattier, nous avons utilisé un milieu de base développé par Alcaraz et collaborateurs (7). Ce milieu contient des éléments minéraux essentiels pour la germination des grains de pollen et la croissance du tube pollinique, comme le 
calcium, le bore et le potassium (7). A ce milieu de base, nous avons ajouté quelques facteurs qui sont connus par leur action stimulateur de la germination du pollen comme les sucres $(4,5,8,11)$, le polyéthylène glycol (PEG) $(7,9,12)$ et l'acide naphtalène acétique (ANA).

Nous avons noté une différence hautement significative dans la germination des grains de pollen entre les différents milieux testés $(\mathrm{P}<0,001)$. Un maximum de germination (91,8 \%) est enregistré avec l'addition du sucrose (Fig.1), alors que pour le témoin, nous avons noté $(66,25 \%)$, et pour le ANA $(23,8 \%)$ (Fig.1). Le faible pourcentage de germination est enregistré pour le PEG (3\%). D'autre part, la germination des grains de pollen est totalement inhibée avec le fructose, le glucose et le jus des dattes (Fig.1).

Les longueurs des tubes polliniques ont suivi la même tendance enregistrée pour la germination (Fig.2). Pour le milieu contenant le sucrose, nos résultats relatifs à la germination du pollen et la croissance des tubes polliniques, sont en accord avec ceux rapportés par plusieurs auteurs $(7,12)$. Notre étude montre aussi que le milieu contenant le fructose, le glucose ou le jus des dattes inhibent la gemination et la croissance du pollen. Pour le glucose, nos résultats sont en désaccord avec les travaux de Okusaka et Hiratsuka (2009) (13), qui ont démontré que le glucose favorise la germination des grains de pollen de Pyrus pyrifolia Nakai. Ces mêmes auteurs ont rapporté que le fructose inhibe la germination du pollen, ce qui est en accord avec nos résultats pour les milieux contenants le fructose ou le jus des dattes, car le jus des dattes contient un taux élevé du glucose et du fructose (14). Chez certaines espèces, l'addition des phytohormones comme les auxines, les cytokinines et les acides gibbérelliques stimule la germination du pollen et la croissance du tube pollinique $(15,16)$. Néanmoins, dans cette étude l'ANA, qu'est une auxine, réduit la germination et la longueur des tubes polliniques en comparaison avec le témoin et le sucrose. Nous avons aussi enregistré une très faible germination avec le PEG. Ces résultats sont en désaccord avec les travaux de Pham et collaborateurs (8) qui ont rapporté l'effet stimulatrice du PEG pour la germination du pollen. Cette différence peut être liée aux différences génétiques entre les espèces. 


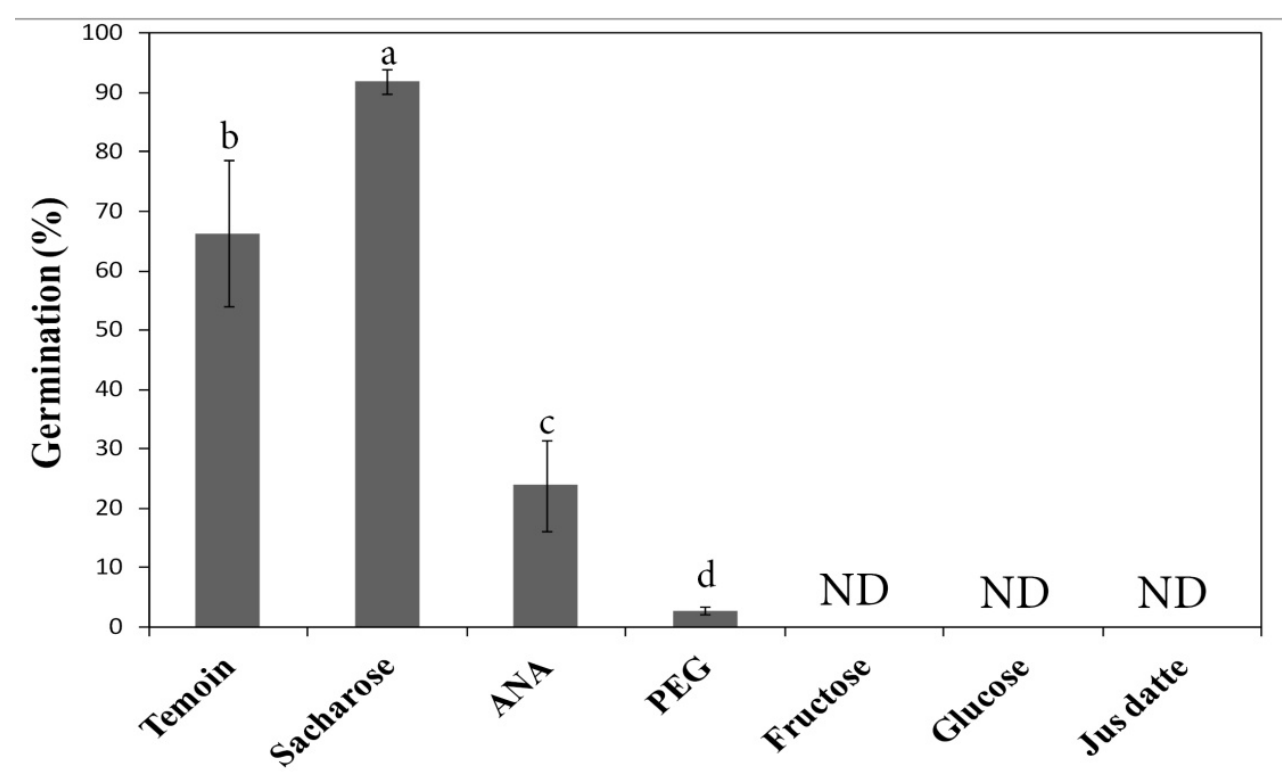

Milieu de culture

Fig.1. Pourcentage de germination des grains de pollen de palmier dattier sous différents milieux de culture, (PEG) polyéthylène-glycol, (ANA) acide naphtalène acétique. Chaque valeur représente la moyenne de six répétitions $\pm \mathrm{SD}$. Pour chaque paramètre, les petites lettres différentes indiquent une différence significative (test de Tukey, $\mathrm{P}<0,05$ ). ND=Non

Determiné.

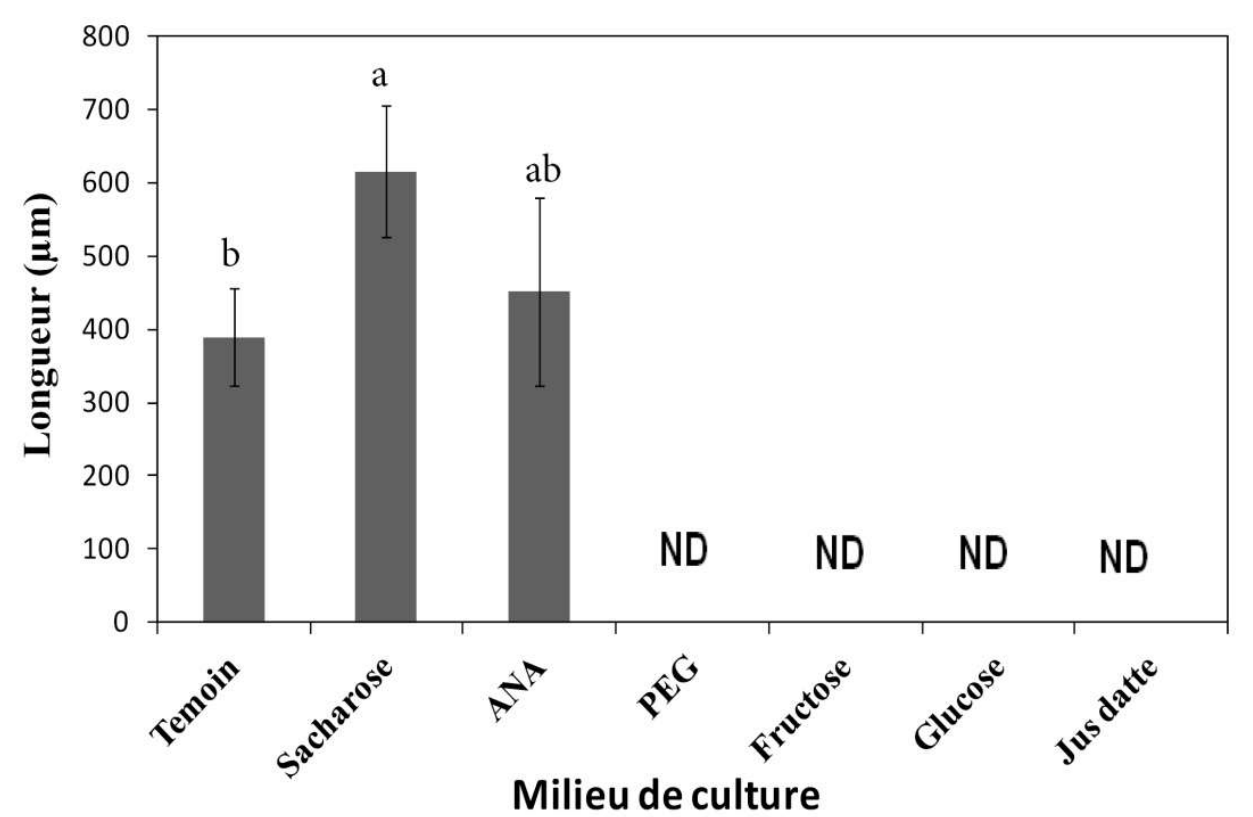

Fig.2. Longueur des tubes pollinique de palmier dattier sous différents milieux de culture, (PEG) polyéthylène-glycol, (ANA) acide naphtalène acétique. Chaque valeur représente la moyenne de vingt répétitions \pm SD. Pour chaque paramètre, les petites lettres différentes indiquent une différence significative (test de Tukey, $\mathrm{P}<0,05$ ). ND=Non Determiné 
Apres définition du milieu optimal pour la germination des grains de pollen contenant le sucrose, nous avons procédé à l'optimisation de la concentration en sucrose. L'analyse de la variance montre que le pourcentage de la germination des grains de pollen augmente significativement avec l'augmentation de la dose du sucrose, ensuite diminue au dessus de $20 \%$ du sucrose $(\mathrm{P}<0,001$; Fig.3).

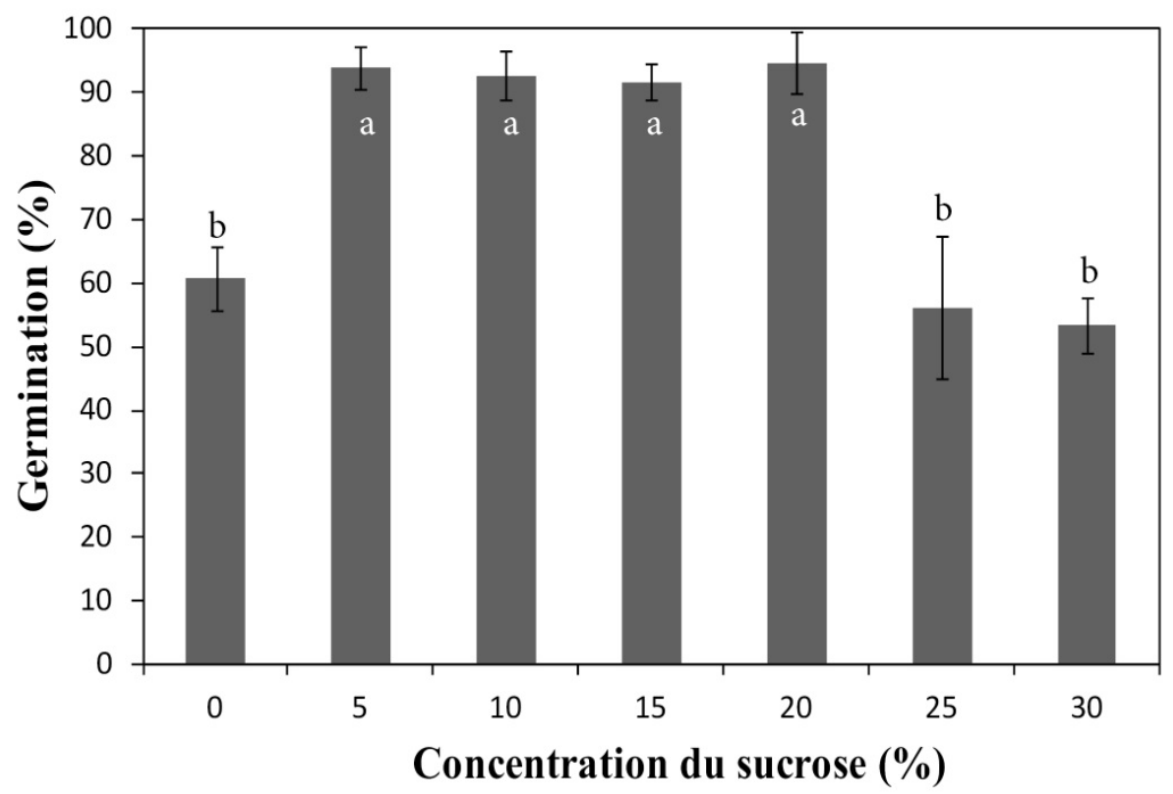

Fig.3. Pourcentage de germination des grains de pollen de palmier dattier sous différents concentrations du sucrose. Chaque valeur représente la moyenne de six répétitions $\pm \mathrm{SD}$. Pour chaque paramètre, les petites lettres différentes indiquent une différence significative (test de Tukey, $\mathrm{P}<0,05)$

Les meilleurs pourcentages de germination (plus de 90\%) sont obtenus entre 5 et $20 \% \mathrm{du}$ sucrose, et sont plus élevé avec ceux rapportés par Mortazavi et al. (2010) pour trois génotypes de palmier dattier, qui ont enregistré environs $80 \%$ de germination. D'autre part la longueur des tubes polliniques est également influencée par la concentration du sucrose, et les tubes les plus longs sont obtenus à $5 \%$ de sucrose (Fig.4 et 5). Les variations de l'effet des différentes concentrations de sucrose peuvent être associées à la variation des potentiels osmotiques (7). D'autres auteurs suggèrent que le saccharose joue un rôle vital à la fois en tant que régulateur osmotique et composé nutritif (8). 


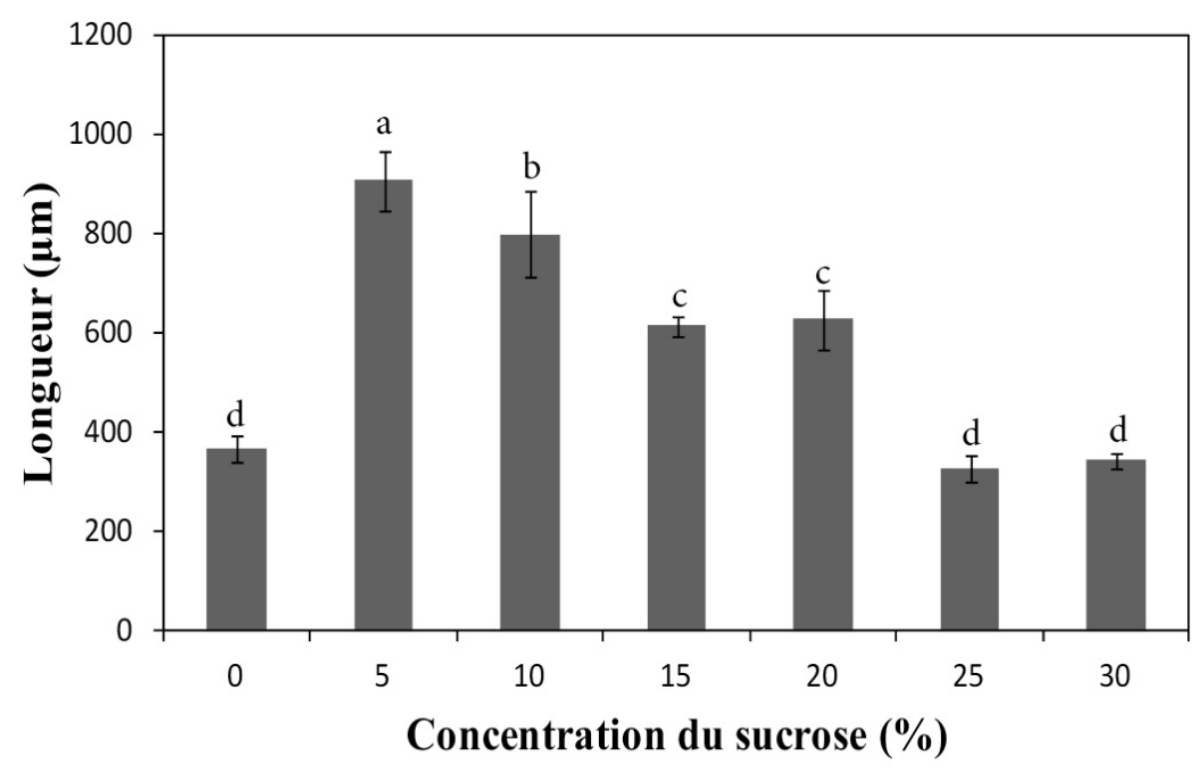

Fig.4. Longueur des tubes pollinique de palmier dattier sous différents concentrations du sucrose. Chaque valeur représente la moyenne de vingt répétitions $\pm \mathrm{SD}$. Pour chaque paramètre, les petites lettres différentes indiquent une différence significative (test de Tukey,

$$
\mathrm{P}<0,05)
$$

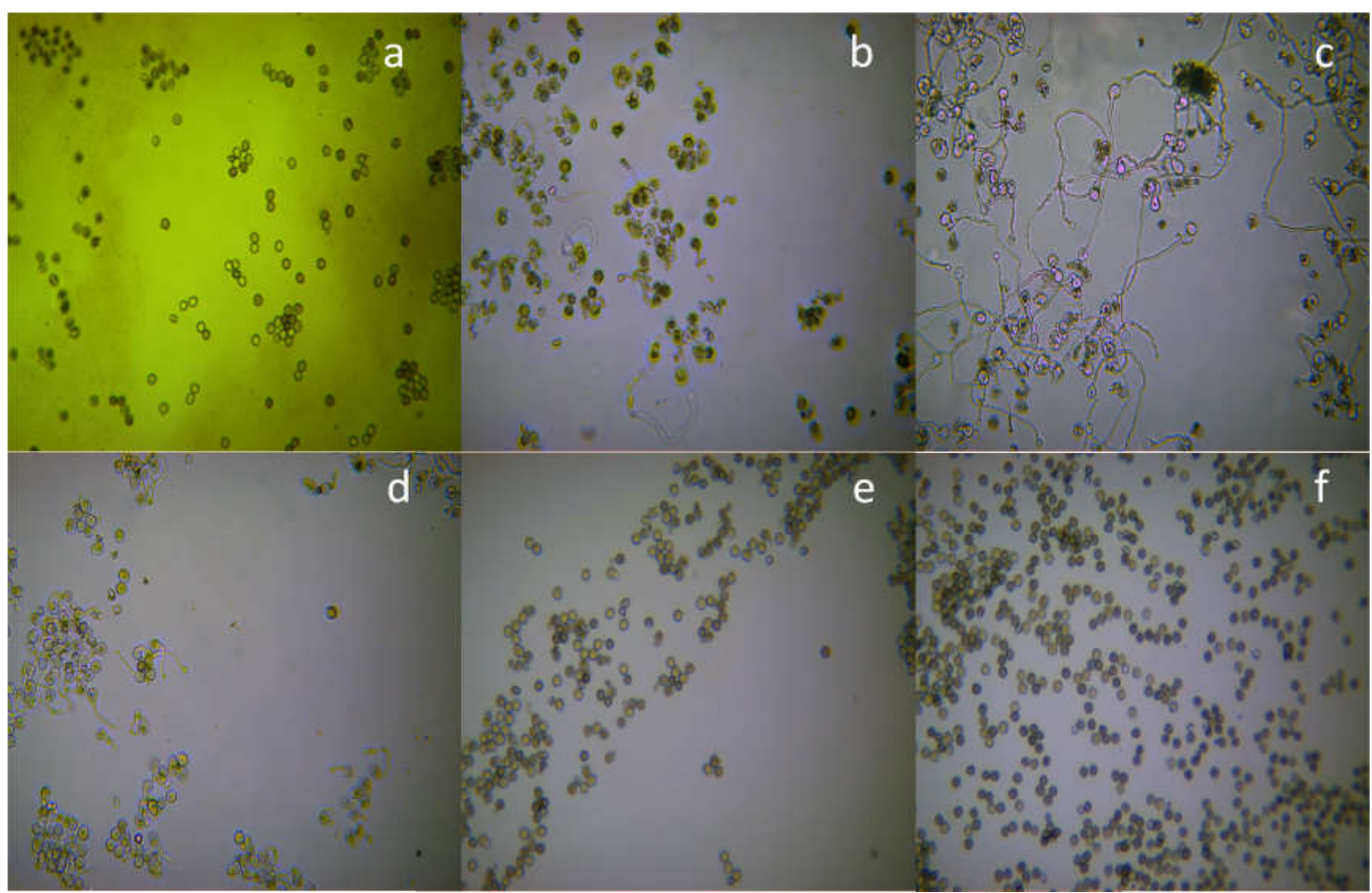

Fig.5. Effet du milieu de culture sur la germination des grains de pollen et les longueurs des tubes polliniques de palmier dattier (a. extrait de datte, b. $0 \%$ du sucrose, c. $5 \%$ du sucrose, d. $30 \%$ du sucrose, e. $20 \%$ glucose, f. $20 \%$ fructose) 


\section{CONCLUSION}

Un milieu de germination du pollen est considéré comme efficace lorsque un maximum de germination, un minimum l'éclatement et une meilleur élongation des tubes polliniques sont obtenus. Selon nos résultats, un milieu contenant $0,42 \mathrm{~g} / \mathrm{l}$ nitrate de calcium, 0,20 $\mathrm{g} / 1$ acide borique, $0,1 \mathrm{~g} / 1$ nitrate de potassium, 0,22 $\mathrm{g} / \mathrm{l}$ sulfate de magnésium et $5 \%$ du sucrose parait optimal pour la germination des grains de pollen et la croissance des tubes polliniques.

En perspective, les résultats de cette étude sont intéressants pour l'étude appliquée de la biologie du pollen pour le palmier dattier.

\section{REFERENCES BIBLIOGRAPHIQUES}

1. Al-Dous EK, George B, Al-Mahmoud ME, Al-jaber MY, Wang H, Salameh YM, Al-azwani EK, Chaluvadi S, Pontaroli AC, Debarry J, et al. De novo genome sequencing and comparative genomics of date palm (Phoenix dactylifera). Nat Biotechnol (2011) 29:521-527. doi:http://dx.doi.org/10.1038/nbt.1860

2. Elleuch M, Besbes S, Roiseux O, Blecker C, Deroanne C, Drira NE, Attia H. Date flesh: Chemical composition and characteristics of the dietary fibre. Food Chem (2008) 111:676-682. doi:10.1016/j.foodchem.2008.04.036

3. FAOSTAT. FAOSTAT Database on Agriculture. FAO - Food Agric Organ United Nations (2015) Available at: http://faostat3.fao.org/home

4. Abdelgadir HA, Johnson SD, Van Staden J. Pollen viability, pollen germination and pollen tube growth in the biofuel seed crop Jatropha curcas (Euphorbiaceae). South African J Bot (2012) 79:132-139. doi:10.1016/j.sajb.2011.10.005

5. Sorkheh K, Shiran B, Rouhi V, Khodambashi M, Wolukau JN, Ercisli S. Response of in vitro pollen germination and pollen tube growth of almond (Prunus dulcis Mill.) to temperature, polyamines and polyamine synthesis inhibitor. Biochem Syst Ecol 39:749-757. doi:10.1016/j.bse.2011.06.015

6. Lora J, Oteyza MAP de, Fuentetaja P, Hormaza JI. Low temperature storage and in vitro germination of cherimoya (Annona cherimola Mill.) pollen. (2006). doi:10.1016/j.scienta.2005.12.003 
7. Alcaraz ML, Montserrat M, Hormaza JI. In vitro pollen germination in avocado (Persea americana Mill.): Optimization of the method and effect of temperature. Sci Hortic (Amsterdam) (2011) 130:152-156. doi:10.1016/j.scienta.2011.06.030

8. Pham VT, Herrero M, Hormaza JI. Effect of temperature on pollen germination and pollen tube growth in longan (Dimocarpus longan Lour.). Sci Hortic (Amsterdam) (2015) 197:470-475. doi:10.1016/j.scienta.2015.10.007

9. Ćalić D, Devrnja N, Kostić I, Kostić M. Pollen morphology, viability, and germination of Prunus domestica cv. Požegača. Sci Hortic (Amsterdam) (2013) 155:118-122. doi:10.1016/j.scienta.2013.03.017

10. Acar I, Kakani VG. The effects of temperature on in vitro pollen germination and pollen tube growth of Pistacia spp. Sci Hortic (Amsterdam) (2010) 125:569-572. doi:10.1016/j.scienta.2010.04.040

11. Mortazavi SMH, Arzani K, Moini A. Optimizing Storage and In vitro Germination of Date Palm (Phoenix dactylifera) Pollen. J Agric Sci Technol (2010) 12:181-189.

12. Sakhanokho HF, Rajasekaran K. Pollen biology of ornamental ginger (Hedychium spp. J. Koenig). Sci Hortic (Amsterdam) (2010) 125:129-135. doi:10.1016/j.scienta.2009.12.037

13. Okusaka K, Hiratsuka S. Fructose inhibits pear pollen germination on agar medium without loss of viability. Sci Hortic (Amsterdam) (2009) 122:51-55. doi:10.1016/j.scienta.2009.03.024

14. Assirey EAR. Nutritional composition of fruit of 10 date palm (Phoenix dactylifera L.) cultivars grown in Saudi Arabia. J Taibah Univ Sci (2015) 9:75-79. doi:10.1016/j.jtusci.2014.07.002

15. Papenfus HB, Kumari A, Kulkarni MG, Finnie JF, Van Staden J. Smoke-water enhances in vitro pollen germination and tube elongation of three species of Amaryllidaceae. South African J Bot (2014) 90:87-92. doi:10.1016/j.sajb.2013.10.007

16. Singh DP, Jermakow AM, Swain SM. Gibberellins are required for seed development and pollen tube growth in Arabidopsis. Plant Cell (2002) 14:3133-47. doi:10.1105/TPC.003046 


\section{OPTIMISATION DES CONDITIONS DE GERMINATION IN VITRO DU POLLEN DE PALMIER DATTIER (PHOENIX DACTYLIFERA L.)}

\section{RESUME}

La germination du pollen et la croissance du tube pollinique ont un effet important sur la qualité des fruits et la production de semences dans la plupart des espèces végétales. Cette étude a été menée pour déterminer un milieu de culture optimal pour la germination du pollen et la croissance de tube pollinique chez le palmier dattier mâle. Des différences significatives au niveau du pourcentage de germination du pollen et de la croissance des tubes polliniques ont été observées entre les différents milieux de culture. L'utilisation d'un milieu à base de l'agar contenant 5\% de sucrose, 0,20 g/l d'acide borique, 0,42 g/l de nitrate de calcium, $0,1 \mathrm{~g} / 1$ de nitrate de potassium et $0,22 \mathrm{~g} / 1$ de sulfate de magnésium a donné les meilleurs résultats en termes de germination et croissance du tube. Le pourcentage de la germination des grains de pollen a diminué avec du polyéthylène-glycol (PEG) et de l'acide naphtalène acétique (ANA), et a été inhibé complètement avec le fructose, le glucose et l'extrait de datte. Ces résultats pourraient avoir des implications non seulement sur l'optimisation de la gestion du pollen chez le palmier dattier, mais aussi dans le choix du meilleur pollinisateur pour cette espèce.

Mots clés: Germination in vitro du pollen; Saccharose; Fructose; Glucose; Acide naphtalèneacétique; Milieu de base.

\section{How to cite this article:}

Mesnoua M, Roumani M, Bensalah MK, Salem A, Benaziza A. Optimization of conditions for in vitro pollen germination and pollen tube growth of date palm (phoenix dactylifera 1.). J. Fundam. Appl. Sci., 2018, 10(1), 158-167. 